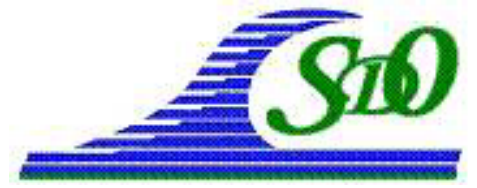

XI ${ }^{\text {èmes }}$ Journées Nationales Génie Côtier - Génie Civil

Les Sables d'Olonne, 22-25 juin 2010

DOI:10.5150/jngcgc.2010.079-K C Editions Paralia CFL

disponible en ligne - http://www.paralia.fr - available online

\title{
Utilisation des bétons fibrés à ultrahautes performances en site portuaire
}

\section{Thierry KUBWIMANA ${ }^{1}$, Nicolas BOURNETON ${ }^{1}$, Nicolas ROUXEL ${ }^{2}$ Aldéric HAUCHECORNE ${ }^{3}$}

1. Cete de l'Ouest, Division Infrastructure et Environnement, Groupe Ouvrage d'Art, MAN, Rue René Viviani, BP 46223, 4426 Nantes cedex 2, France.

thierry.kubwimana@bvra.entpe.fr

nicolas.bourneton@developpement-durable.gouv.fr

2. Cete de l'Ouest, Laboratoire Régional des Ponts et Chaussées de St-Brieuc, Equipe de Recherche Associée au CETMEF «Durabilité des Infrastructures Portuaires et Maritimes, 5 rue Jules Vallès, 22015 Saint Brieuc Cedex, France.

nicolas.rouxel@developpement-durable.gouv.fr

3. CETMEF, Division Ouvrages Portuaires et Maritimes,

2 Boulevard Gambetta, BP60039, 60321 Compiègne Cedex, France.

alderic.hauchecorne@developpement-durable.gouv.fr

\section{Résumé :}

Employé depuis le milieu des années 1990, le BFUP a montré de nouvelles possibilités dans la conception de structures dans le génie civil et le bâtiment ainsi que dans réhabilitation d'ouvrages anciens. Cette étude, réalisée à la demande du CETMEF a pour but de définir l'opportunité d'une solution en BFUP pour la réalisation d'un quai. L'étude a ainsi porté sur le dimensionnement d'une alternative BFUP à un ouvrage de référence : le quai en eaux profondes de Kergroise (Lorient) qui est un quai béton sur pieux métalliques. Seules les sollicitations mécaniques principales de l'ouvrage de référence ont été retenues pour le dimensionnement. Après avoir présenté les utilisations du matériau, ses caractéristiques, et le cadre réglementaire, plusieurs solutions ont été envisagées et dimensionnées.

Dans un premier temps, il est apparu que malgré le gain de matière, la simple substitution du béton armé par du BFUP ne suffit pas pour rendre cette solution économiquement compétitive. Une solution de BFUP précontraint a alors été étudiée en augmentant la portée des poutres c'est-à-dire en réduisant le nombre de pieux. Des considérations de mise en œuvre de cette précontrainte ont conduit à revoir le fonctionnement de l'ouvrage: l'option BFUP finalement retenue est une structure isostatique dans laquelle le maillage a été desserré, permettant ainsi de diminuer significativement le nombre de pieux nécessaires sous l'ouvrage.

Dans ces conditions, la solution BFUP a été comparée à la solution en béton armé selon les critères suivants : coût, consommation d'énergie et rejet de gaz à effet de serre.

Mots-clés : Quai - BFUP - Béton armé - Analyse des coûts - Environnement 


\section{Introduction}

Le présent article résume une étude sur l'opportunité d'utiliser du béton fibré ultrahautes performances (BFUP) pour la réalisation d'ouvrages portuaires. L'étude complète est mise a disposition par le CETMEF (www.cetmef.developpementdurable.gouv.fr). Il s'agit, au travers de l'exemple du quai en eaux profondes de Lorient, de montrer les intérêts et inconvénients d'un point de vue technique et économique d'une solution en BFUP par rapport à une solution béton armé. La démarche a été de dimensionner l'ouvrage en considérant uniquement l'aspect mécanique, puis de mettre en avant les avantages ou inconvénients collatéraux à l'utilisation du BFUP.

L'ouvrage de référence est un quai de type dalle et poutres, surmonté d'un remblai général, sur fondations profondes par pieux métalliques. La plateforme est de largeur variable : large de 31,51 m en section courante, elle présente une zone d'élargissement au sud ainsi qu'un poste de stationnement, voir figure 1. Sur une même file, les pieux sont espacés de $5 \mathrm{~m}$.

Le platelage du quai est constitué de poutres préfabriquées posées sur des têtes de pieux préfabriquées ; les pieux sont bétonnés sur une hauteur de $3 \mathrm{~m}$ afin de pouvoir réaliser un chapiteau en béton au niveau de la tête.

La continuité mécanique des poutres est assurée par le clavage entre celles-ci au niveau des pieux par le coulage en place de la partie supérieure des poutres longitudinales qui doivent assurer la continuité des rails de grues entre le quai existant et le quai projeté.

De même la poutre de front de quai est constituée d'une partie préfabriquée et d'une partie coulée en place.

Les dalles sont réalisées dans un premier temps avec des prédalles posées sur les poutres préfabriquées puis finalisées lors du coulage en place du complément de béton assurant la liaison des poutres avec le platelage.

Lors de l'appel d'offre, l'estimation des travaux était de l'ordre de $7865 \mathrm{k} €$ pour la réalisation de l'extension sud.

\section{Variante BFUP}

\subsection{Principes de calcul}

Le calcul des efforts dans les poutres et les pieux a été réalisés dans un premier temps grâce à une modélisation complète de la poutraison sous ST1 (calculateur de structures à barres développé par le SETRA). Les pieux ont été modélisés comme étant parfaitement encastrés en pied.

Le logiciel ST1 ne permettant pas la définition d'éléments surfaciques, le calcul des dalles est effectué séparément sur le logiciel ESA-Prima Win (logiciel de calcul éléments finis développé par la société SCIA). La vérification des sections est effectuée sous INCA 2. Ce logiciel développé par 1'Université de Hambourg permet d'obtenir la réponse en flexion de tout type de section. 
A noter enfin que le rideau de soutènement arrière n'est pas redimensionné. Pour le dimensionnement on retient les efforts qui ont servi à calculer la solution béton armé. Il faut noter que seules les sollicitations mécaniques principales de l'ouvrage de référence ont été retenues pour le dimensionnement

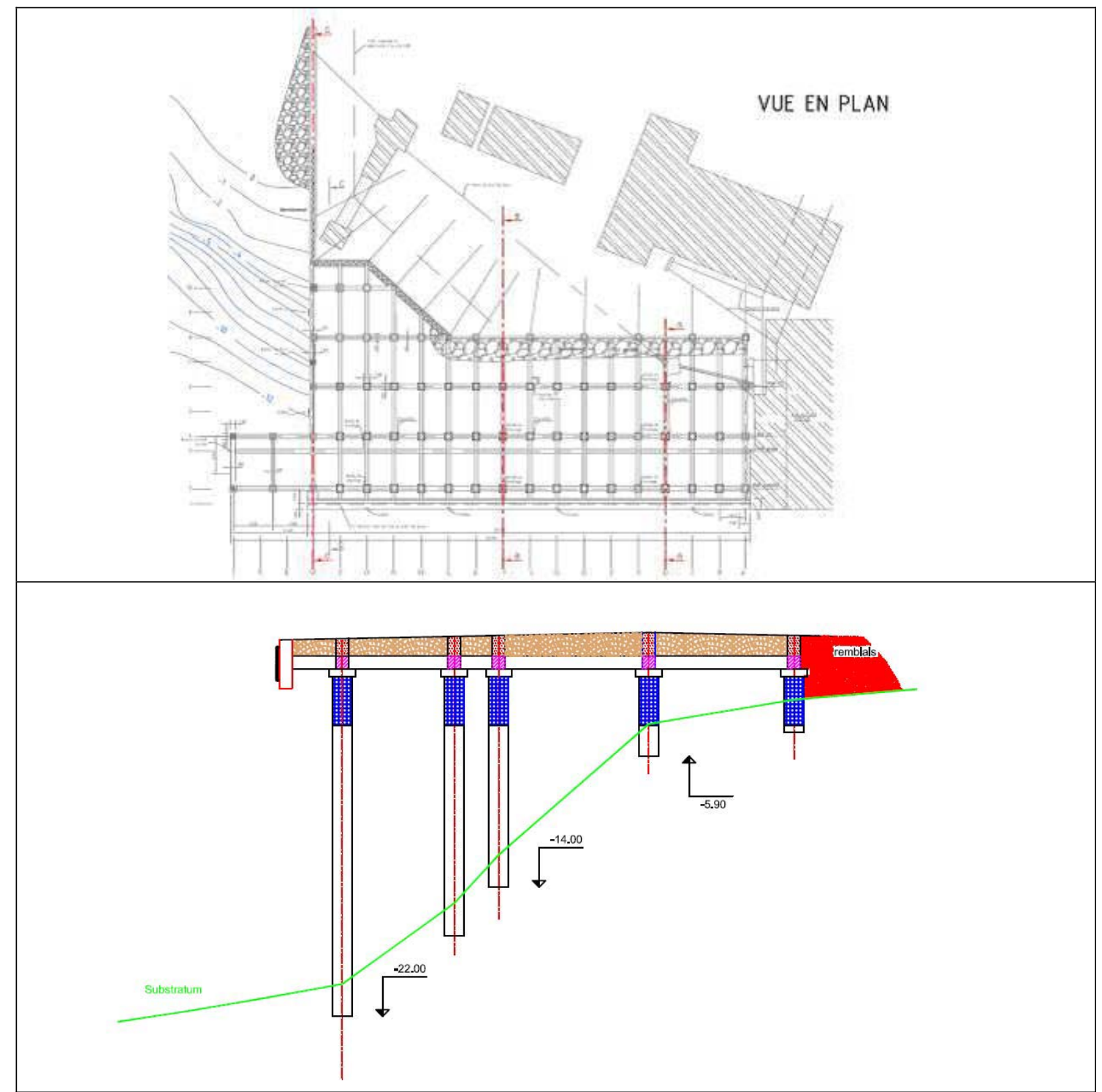

Figure 1. Vue en plan et coupe de principe de la solution de référence en béton armé.

\subsection{Matériaux}

Le BFUP

Pour le dimensionnement, nous avons opté pour un très bon BFUP ayant subi un traitement thermique dont les caractéristiques sont reprises dans le tableau ci dessous. Les valeurs entre parenthèses sont des valeurs caractéristiques d'un BFUP de classe moyenne. 
Thème 5 - Ouvrages portuaires, côtiers et offshore

Tableau 1. Caractéristiques du BFUP pris en compte dans l'étude.

$\begin{array}{ll}\begin{array}{l}\text { Résistance caractéristique à la compression à } 28 \\ \text { jours } f_{\text {c28 }}\end{array} & 200 \mathrm{MPa}(180 \mathrm{MPa}) \\ \begin{array}{l}\text { Résistance caractéristique à la traction directe à } \\ 28 \text { jours } f_{\text {t28 }}\end{array} & 9 \mathrm{MPa}(9 \mathrm{MPa}) \\ \begin{array}{l}\text { Résistance caractéristique du matériau fissuré } \sigma_{b t} \\ \text { Module d'élasticité E }\end{array} & 55 \mathrm{MPa}(9 \mathrm{MPa}) \\ \text { Coefficient de Poisson } & 0,2(50 \mathrm{GPa}) \\ \text { Coefficient de dilatation thermique } & 1,1 \times 10^{-5} \mathrm{~m} / \mathrm{m} /{ }^{\circ} \mathrm{C}\left(1,1.10^{-5} \mathrm{~m} / \mathrm{m} /{ }^{\circ} \mathrm{C}\right) \\ \text { Retrait total } & 550 \mu \mathrm{m} / \mathrm{m}(550 \mu \mathrm{m} / \mathrm{m}) \\ \text { Fluage } & 0,2(0,2)\end{array}$

Les éléments en BFUP sont calculés conformément au document AFGC (2002) les hypothèses suivantes :

- isotropie dans la répartition des fibres, soit un coefficient de dispersion global $\mathrm{K}=1,25$,

- taux minimal de fibres vérifié,

- loi de comportement écrouissante,

- pièces justifiées en classe II (une traction de ftj sera admise sous l'effet des combinaisons rares),

- mêmes justifications pour les sections de reprises,

- déflexion maximale de $1 \mathrm{~cm}$.

Les justifications sous contraintes normales à l'ELS rares sont analogues à celles du BPEL : les contraintes de compression sont limitées à $0,6 \mathrm{f}_{\mathrm{c} 28}$ et la traction est limitée à $\mathrm{f}_{\mathrm{t} 28 .}$.

A l'ELU, le moment résistant est calculé via INCA 2 en prenant en compte la loi de comportement du matériau fissuré.

Enfin, les vérifications des contraintes de cisaillement à l'ELS sont conduites de manière analogue aux spécifications du BPEL.

\section{Les aciers}

Pour les aciers de précontrainte, on utilise des torons T15 S de classe $1860 \mathrm{MPa}$.

L'acier utilisé pour les pieux est le même que pour la solution armée, à savoir un acier $\mathrm{X} 60$ tel que fe $=420 \mathrm{MPa}$.

L'acier utilisé pour les éléments de structure est un acier S 355 tel que fe $=355 \mathrm{MPa}$. 


\section{XI $I^{\text {èes }}$ Journées Nationales Génie Côtier - Génie Civil}

Les Sables d'Olonne, 22-25 juin 2010

\subsection{Principaux résultats}

Toutes les étapes de calculs ainsi que les plans précis sont rappelés dans le rapport CETMEF (2010).

Les principales modifications par rapport à la structure en béton armé de référence sont :

- la poutraison est réalisée avec des poutres BFUP en I plus ou moins précontraintes (10T15 à 20T15) dont les dimensions sont présentées sur les figures 2 et 3 ,

- sur une même file de pieux, l'espacement entre deux pieux est augmenté,

- une file de pieux sous une voie de grue est rajoutée.

- les dalles sont de structure mixte BFUP - Béton.

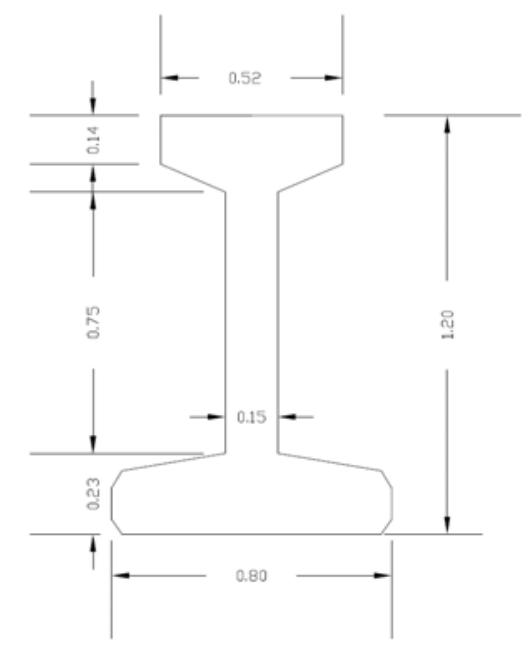

Figure 2. Coupe type des poutres BFUP.

\section{Analyse multicritères des deux variantes}

\section{$3.1 \underline{\text { Coût }}$}

Malgré le prix légèrement supérieur du BFUP on constate que les deux solutions considérées ont des coûts comparables : $7850 \mathrm{k} €$ pour l'ouvrage en béton armé contre $8050 \mathrm{k} €$ pour l'option BFUP. Le surcoût dû à l'utilisation de BFUP est compensé par l'économie du nombre de pieux que permet l'emploi des poutres précontraintes en BFUP.

\subsection{Mise en œuvre}

Les deux options, BFUP et béton armé, prévoient l'emploi de préfabrication. L'option BFUP va ainsi nécessiter la réalisation de moins de pièces du fait de l'allongement de la portée des poutres, mais celles-ci vont faire l'objet d'un contrôle plus strict. Les poutres préfabriquées BFUP sont aussi légèrement plus lourdes que les poutres préfabriquées de l'ouvrage de référence. 


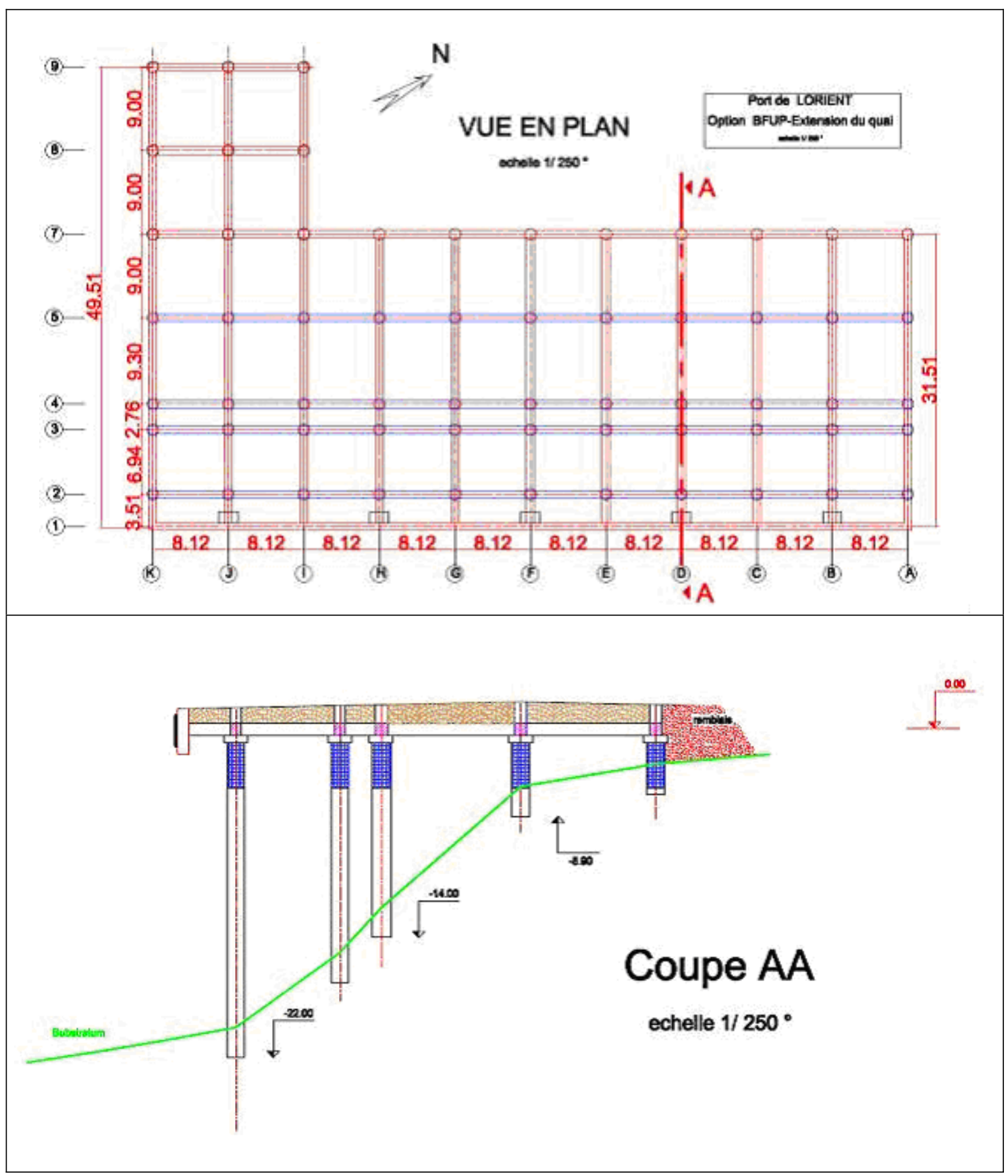

Figure 3. Vue en plan et coupe de principe de la solution BFUP.

\subsection{Durabilité}

Bien que le BFUP ait des propriétés bien plus remarquables qu'un béton ordinaire en termes de durabilité, ceci ne suffit pas à garantir, a priori, une durée de vie d'ouvrage accrue : les pieux, communs aux deux solutions, vont être le facteur limitant. En théorie, l'ouvrage en BFUP ne devient plus durable que moyennant la prise de mesures plus 
efficaces pour la protection des pieux (ex : protection cathodique, épaisseur sacrificielle plus importante) en début de projet.

\subsection{Surveillance et entretien}

Les méthodes de gestion ne vont pas varier d'un ouvrage à l'autre : aucun ne requiert un entretien ni un niveau de surveillance spécifique malgré le peu de retour d'expériences sur l'utilisation de BFUP précontraint en site maritime.

\subsection{Impact environnemental}

A ce stade du projet il est difficile d'évaluer pertinemment les émissions durant les différentes étapes de la vie de l'ouvrage (fabrication, transport, utilisation en service, renforcement, destruction...). On se limite donc à l'étude de la phase fabrication - étant couramment la phase dominante - c'est-à-dire au sortir de la centrale de béton prêt à l'emploi au nord de Lorient.

Depuis 2004, la base INIES (www.inies.fr) met librement à disposition les caractéristiques environnementales de certains produits de construction conformément à la norme NF P01-10 (Qualité environnementale des produits de construction Déclaration environnementale et sanitaire des produits de construction). Raisonnant sur des unités fonctionnelles précises, nous avons rapporté ces données au $\mathrm{m}^{3}$ de matériau et, utilisé les FDES (Fiches de Déclaration Environnementale et Sanitaire) se rapprochant au plus près des matériaux considérés pour les deux variantes.

Comme il n'existe pas encore de FDES se rapprochant du BFUP, l'impact environnemental a été calculé en suivant la démarche proposée par BOUHAYA \& LE ROY (2008) à savoir sommer l'impact environnemental de chaque constituant pris séparément, voir le tableau 2 .

Connaissant les caractéristiques environnementales du BFUP, d'un béton classique et de l'acier, on calcule l'impact environnemental des deux solutions.

Grâce à la diminution du tonnage d'acier nécessaire combiné au gain de matière, on observe que la consommation d'énergie ainsi que les émissions de gaz à effets de serre sont divisées par deux : la solution BFUP est pertinente pour l'environnement.

Il convient cependant de relativiser la pertinence des valeurs ci-dessus. On rappelle en effet que ces chiffres prennent en compte uniquement la production des matériaux. Au stade de la préfabrication, le traitement thermique que peuvent subir les poutres BFUP est susceptible de réduire cet écart.

Tableau 2. Impact environnemental de chaque matériau.

\begin{tabular}{|c|c|c|c|}
\hline & Béton ordinaire & Acier & BFUP \\
\hline Unité fonctionnelle & Poutre en béton $B A P$ & Poutrelle en acier & Sans objet \\
\hline Energie non renouvelable & $3,6 \mathrm{GJ} / \mathrm{m}^{3}$ & $150 \mathrm{GJ} / \mathrm{m}^{3}$ & $8,6 \mathrm{GJ} / \mathrm{m}^{3}$ \\
\hline Emission de GES & 0,42 téq $\mathrm{CO} 2 / \mathrm{m}^{3}$ & 10,4 téq $C O 2 / m^{3}$ & 1,1 téq $\mathrm{CO} 2 / \mathrm{m}^{3}$ \\
\hline
\end{tabular}



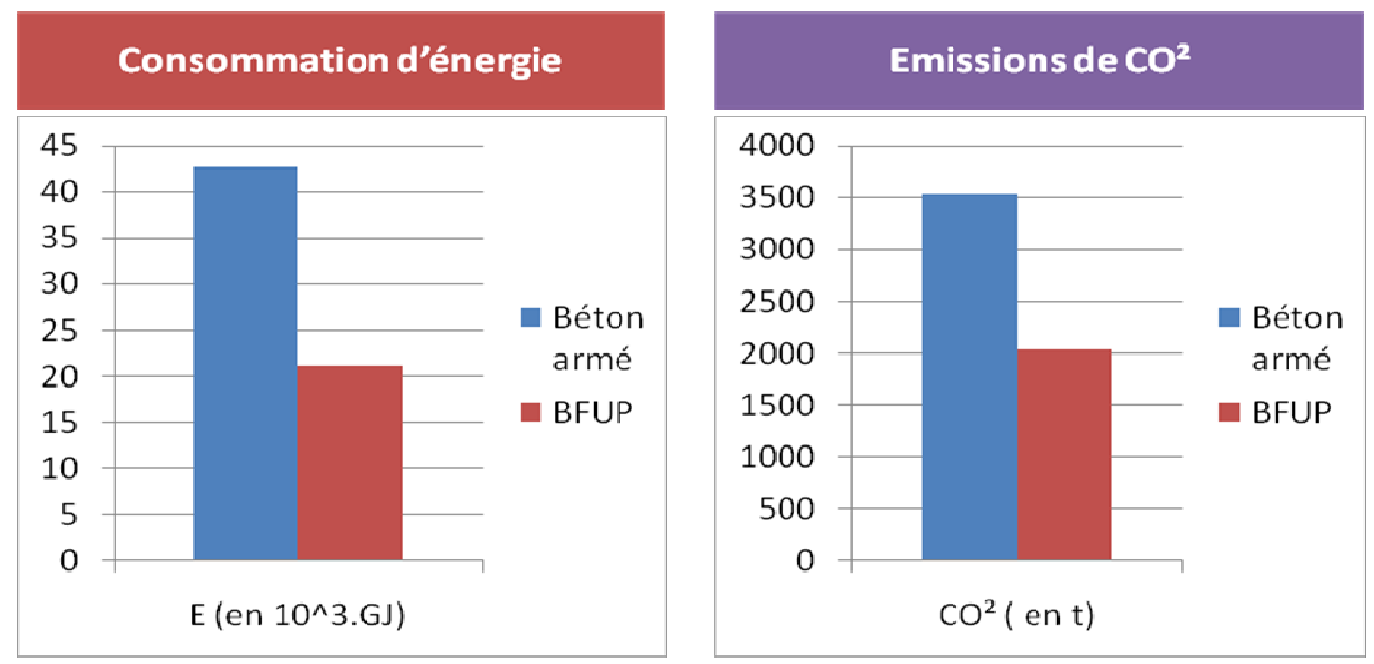

Figure 4. Impact environnemental des deux solutions.

\section{Conclusion et perspectives}

Avec l'expérience acquise depuis les premiers ouvrages en BFUP, il est apparu raisonnable d'envisager la réalisation d'un quai sur pieux. La solution alternative en BFUP pour le projet d'extension sud des quais de Kergroise s'est montré légèrement plus onéreuse $(+3 \%)$ mais plus innovante et compétitive sous plusieurs aspects :

- réduction du nombre de pièces à préfabriquer ainsi que la diminution du nombre de pieux.

- La durée de vie de l'ouvrage est limitée par la durée de vie des éléments métalliques. Si la corrosion des pieux est correctement prise en compte (épaisseur sacrificielle, protection cathodique...), la durabilité du quai est améliorée.

- La consommation d'énergie et les rejets de gaz à effets de serre sont a priori moins élevés pour la solution BFUP que pour la solution en béton armé de référence.

Cette étude montre ainsi qu'une solution en BFUP est envisageable pour la réalisation d'un quai sur pieux. La réalisation d'un quai entièrement en BFUP (pieux compris) permettrait de construire un ouvrage dont la durée de vie est nettement supérieure à celle de l'ouvrage de référence en béton armé. La faisabilité et l'intérêt d'un tel ouvrage reste à établir.

\section{Références bibliographiques}

AFGC (2002). Bétons fibrés ultra-hautes performances - Recommandations provisoires. AFGC-SETRA, janvier 2002.

CETMEF (2010). Utilisation des bétons fibrés à ultra hautes performances en site maritime : Port de Lorient-Quai de Kergroise. Rapport.

BOUHAYA L., LE ROY R. (2008). Analyse du cycle de vie des ponts de moyenne portée: les apports du matériau bois. Présentation aux Journées Ouvrages d'Art, Bordeaux, Mai. 